\title{
Modified Cooperative Subchannel Allocation Algorithms and PSO Based Power Allocation for an Alamouti Decode and Forward Relaying Protocol in Multiuser OFDMA Systems
}

\author{
K. Shoukath Ali, P. Sampath \\ Department of ECE, Bannari Amman Institute of Technology, Sathyamangalam, India \\ Email: shoukathali@bitsathy.ac.in, sampathpalaniswami@gmail.com
}

Received 21 March 2016; accepted 15 April 2016; published 20 June 2016

Copyright (C) 2016 by authors and Scientific Research Publishing Inc.

This work is licensed under the Creative Commons Attribution International License (CC BY).

http://creativecommons.org/licenses/by/4.0/

(c) (i)

\section{Abstract}

The present work is a discussion on the performance analysis of Modified Cooperative Subchannel Allocation (CSA) Algorithms which is used in Alamouti Decoded and Forward (Alamouti DF) Relaying Protocol for wireless multi-user Orthogonal Frequency Division Multiplexing Access (OFDMA) systems. In addition, the performance of approximate Symbol Error Rate (SER) for the Alamouti DF Relaying Protocol with the Cooperative Maximum Ratio Combining Technique (C-MRC) is analyzed and compared with SER upper bound. The approximate SER is asymptotically tight bound at higher Signal-to-Noise Ratio (SNR). From the asymptotic tight bound approximate SER, Particle Swarm Optimization (PSO) based Power Allocation (PA) is determined for the Alamouti DF Relaying Protocol. The simulation results suggested that the Modified Throughput based Subchannel Allocation Algorithm achieved an improved throughput of $6 \%$ to $33 \%$ compared to that of existing cooperative diversity protocol. Further, the Modified Fairness based Subchannel Allocation Algorithm rendered fairness of $7.2 \%$ to $17 \%$ among the multiuser against the existing cooperative diversity protocol.

\section{Keywords}

Amplify-and-Forward, Decode-and-Forward, Cooperative Relay, Maximal Ratio Combining, Symbol Error Rate 


\section{Introduction}

The random fading nature of the wireless communication channel has attracted many researchers to propose novel techniques to raise the cooperative diversity order [1] [2]. To arrive at the diversity gain, a cooperative technique in combination with relaying terminal is used [3] [4]. Of the several known methods of communication using a MIMO system, Alamouti coding scheme is the well characterized approach to adopt the high speed and consistent wireless communication system. Improved bit error rate as well as high data rate can be achieved by exploiting diversity gain in the spatial domain [5]-[7]. In wireless communication systems, the Cooperative relaying protocol promises a considerable capacity and increased multiplexing gain [8] [9]. Cooperative communication is mainly classified into two relaying protocol: Amplify-and-Forward (AF) Relaying Protocol and Decode-and-Forward (DF) Relaying Protocol. AF Relaying Protocol amplifies the Relay Station (RS) information which is received from the Base Station (BS) and is retransmitted to the Mobile Station (MS). The pros of the AF Relaying Protocol are its low cost implementation and simplicity. However, the drawback of the above relaying protocol is that the noise also gets amplified at the RS. In contrast to AF Relaying Protocol, DF Relaying Protocol decodes the RS information received from the BS and then retransmits the re-encoded information to the MS. The signal received from the BS and RS at the MS will be combined using a Maximal Combining Ratio (MRC) technique [10]. Unlike AF Relaying Protocol, the noise is rectified and hence, the DF Relaying Protocol usually shows a better transmission performance than the AF Relaying Protocol.

Previously, it has been reported that the performance analysis of SER upper-bound for Alamouti-coded DF Relaying Protocol used the C-MRC technique along with an optimum PA [11] [12]. The concept that the equal PA and PSO PA do not depend on weighting factor $W$, has not been discussed in [13] [14]. The C-MRC signal combining technique introduces a weighting factor, and it can be considered by value of the channel. The CMRC technique guarantees the enhance throughput of the system and maximum SNR.

In this paper, the performance of approximate SER for the Alamouti DF Relaying Protocol using C-MRC is analyzed, and compared with SER upper bound. In this, the approximate SER is asymptotically tight bound at higher SNR. From the asymptotic tight bound approximate SER, PSO based PA is determined for the Alamouti DF Relaying Protocol. The link between base station and mobile station contributes diversity order one in the system performance, and also depends on the balance of the two channel links from the base station to the relay station and from the relay station to the mobile station. Therefore, the Alamouti DF Relaying Protocol for wireless systems shows an overall performance of diversity orders two.

To achieve high data rates in mobile communication environment, OFDM is a hopeful method because of its multicarrier modulation technique [15]-[17]. In the selected literatures, downlink of non cooperative multi-users OFDMA systems is described using the dynamic Resource Allocation Algorithms (RAA) and optimization techniques. The aim of the RAA is either to achieve the constrained total transmits power with highest throughput and the minimum total transmitted power with constrained throughput [18]-[22]. Cooperative subcarrier allocation for AF Relaying Protocol gives the increased throughput and fairness among the multi-user OFDMA systems. Cooperative subcarrier allocation for AF Relaying Protocol is used EPA, which does not provide enhanced throughput and optimized power to wireless systems. The MS is combined with the signal that is received from the BS and RS using MRC technique. Though, there exist limitations in MRC technique cannot assure the maximum SNR and improved throughput as mentioned in [23] [24].

In addition, in this paper the performance analysis of proposed modified CSA Algorithms used in Alamouti DF Relaying Protocol for wireless multi-user OFDMA systems so as to achieve increased throughput than the existing relaying protocols. The modified CSA Algorithms used in Alamouti DF Relaying for PSO based PA gives the enhanced throughput and optimized wireless systems.

The rest of this paper is organized as the following: in Section 2, received signal model is described. Performance analysis of Alamouti DF Relaying Protocol is discussed in Section 3. In Section 4, Modified Cooperative Subchannel Allocation for an Alamouti DF Relaying Protocol is presented. The simulation results are discussed in Section 5 and Section 6 concludes this research work.

\section{Received Signal Model}

In this paper, system model consist of one BS, two RS and one MS for an Alamouti DF Relaying Protocol in wireless multi-user OFDMA systems is shown in Figure 1 [11].

Orthogonal Space Time Block Codes (OSTBC) is using multiple transmit and receive antenna, which gives 


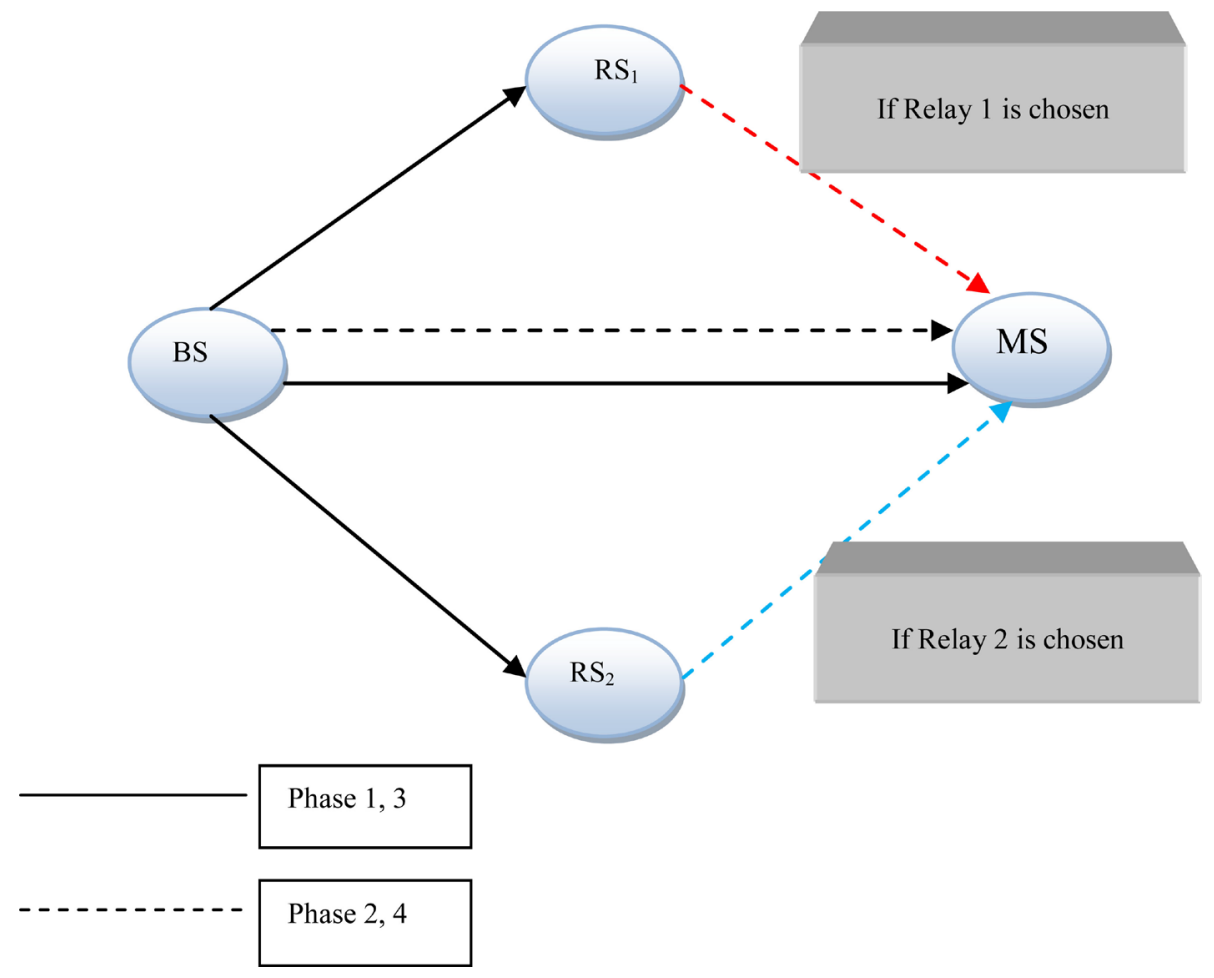

Figure 1. System model for cooperative relaying protocols.

full data rate and full diversity gain to the system. Alamouti scheme is used to enhance the quality of the channel in the received signal. The encoder transmit matrix takes two modulated symbol $X_{1}$ and $X_{2}$ at a time. This is described as,

$$
X=\left(\begin{array}{cc}
X_{1} & X_{2} \\
-X_{2}^{*} & X_{1}^{*}
\end{array}\right)
$$

where $X^{*}$ complex conjugate of $X$. It is clear that the encoding process is done in both space and time domain [25]. The TDMA transmission for Alamouti DF Relaying Protocol is divided into four phases and it is shown in Figure 2. In phase 1, BS is transmitting its information to selected RS by optimum relay selection technique. In phase 2, the transmitted information from BS is decoded using selective RS then forwarded to MS based on Alamouti scheme. Similarly, the process carried out in phase 3 and 4 is same as phase 1 and 2 respectively.

In phase 1, the BS is transmitting information to RS and MS. Let us consider, $Y_{(b-m) 1}, Y_{(b-r) 1}$ are the received signals at RS and MS respectively. Then, it is described as follows:

$$
\begin{gathered}
Y_{(b-m) 1}=\sqrt{P_{1}} h_{(b-m) 1} X_{1}+n_{(b-m) 1} \\
Y_{(B-R) 1}=\sqrt{P_{1}} H_{(B-R) 1} X_{1}+n_{(B-R) 1}
\end{gathered}
$$

In phase 2, the MS receives information from RS and BS is gives as,

$$
Y_{m 2}=\sqrt{W P_{1}} h_{(b-m) 2} X_{2}+\sqrt{W P_{2}} h_{(r-m) 2} \hat{X}_{1}+n_{m 2}
$$

Assume, the BS is transmitting a symbol $X_{1}$ to MS and RS with transmitting power of $P_{1}$ in phase1. $Y_{m 2}$ is the received signal in phase 2, in which the BS are transmitting a symbol $X_{2}$ and the RS is able to decodes the correct symbol and forwards the decoded symbol $\hat{X}_{1}$ to MS with transmitting power of $P_{1}$ and $P_{2}$ respectively. Similarly, $Y_{(b-m) 3}$ and $Y_{(b-r) 3}$ are the received signals in phase 3 with transmitting symbol of $-X_{2}^{*}$ and transmitting power of $P_{1}$. Then, the BS is transmitting a symbol $X_{1}^{*}$ and the RS is able to decode the correct symbol and forwards the decoded symbol $-X_{2}^{*}$ to MS with transmitting power of $P_{1}$ and $P_{2}$ respectively in phase 4. The received signals in phase 3 and 4 are described in Equations (5)-(7). 

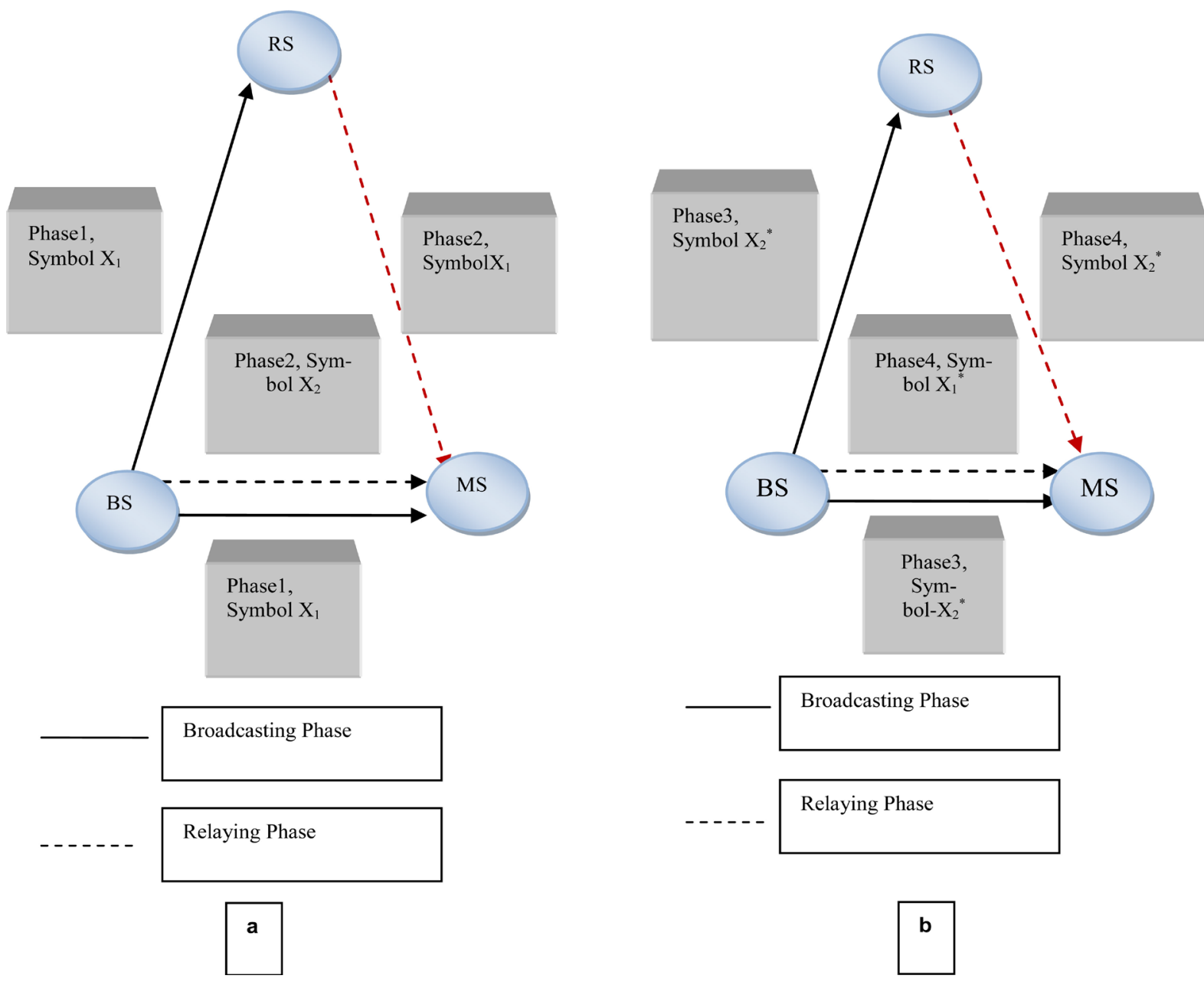

Figure 2. Alamouti DF relaying protocol in different phases.

$$
\begin{gathered}
Y_{(b-m) 3}=\sqrt{P_{1}} h_{(b-m) 3}\left(-X_{2}^{*}\right)+n_{(b-m) 3} \\
Y_{(b-r) 3}=\sqrt{P_{1}} h_{(b-r) 3}\left(-X_{2}^{*}\right)+n_{(b-r) 3} \\
Y_{m 4}=\sqrt{W P_{1}} h_{(b-m) 4} X_{1}^{*}+\sqrt{W P_{2}} h_{(r-m) 4}\left(-\widehat{X}_{2}^{*}\right)+n_{m 4}
\end{gathered}
$$

where $h_{(b-m) 1}, h_{(b-r) 1}, h_{(b-m) 2}$, and $h_{(r-m) 2}$ are the channel coefficient of the BS-to-MS in phase 1, the BS-to$\mathrm{RS}$ in phase 1, the BS-to-MS in phase 2, and the RS-to-MS in phase 2 respectively. Also, $n_{(b-m) 1}, n_{(b-r) 1}, n_{m 2}$ is the additive noise in phase 1 and phase 2.

The total SNR at the MS using C-MRC technique as follows:

$$
\Gamma_{\text {Total }}=\Gamma_{(b-m)}+\Gamma_{\text {Alamouti }}
$$

where $\Gamma_{\text {Total }}$ is the total SNR received at MS from BS and RS. $\Gamma_{(b-m)}$ is the direct path SNR transmitted from BS to MS in phase 1 or 3, and $\Gamma_{\text {Alamouti }}$ is the indirect path SNR at the MS received from RS in phase 2 or 4.

The C-MRC for the received signals at the MS in Equations (2), (4), (5), and (7) are combined and could be expressed as follows:

$$
\begin{gathered}
y_{\hat{X}_{2}(C M R C)}=W_{21(C M R C)}\left(-Y_{(b-m) 3}^{*}\right)+W_{22(C M R C)}\left(Y_{m 2}\right)+W_{23(C M R C)}\left(-Y_{m 4}^{*}\right) \\
y_{\hat{X}_{1}(C M R C)}=W_{11(C M R C)}\left(Y_{(b-m) 1}\right)+W_{12(C M R C)}\left(Y_{m 2}\right)+W_{13(C M R C)}\left(-Y_{m 4}^{*}\right)
\end{gathered}
$$


where $y_{\hat{X}_{2}(C M R C)}$ and $y_{\hat{X}_{1}(C M R C)}$ are the resultant of the C-MRC combiner at the MS [11] [26]. Also $W_{11(C M R C)}{ }^{-}$ $W_{23(C M R C)}$ are the C-MRC weights and expressed in Equations (11)-(16).

$$
\begin{aligned}
& W_{11(\text { CMRC })}=\frac{\sqrt{P_{1}}}{N_{0}} h_{(b-m) 1}^{*} \\
& W_{12(\text { CMRC })}=\frac{\sqrt{W P_{2}}}{N_{0}} h_{(r-m) 2}^{*} \\
& W_{13(\text { CMRC })}=\frac{\sqrt{W P_{1}}}{N_{0}} h_{(b-m) 4} \\
& W_{21(\text { CMRC })}=\frac{\sqrt{P_{1}}}{N_{0}} h_{(b-m) 3}^{*} \\
& W_{22(\text { CMRC })}=\frac{\sqrt{W P_{1}}}{N_{0}} h_{(b-m) 2}^{*} \\
& W_{23(\text { CMRC })}=\frac{\sqrt{W P_{2}}}{N_{0}} h_{(r-m) 4}
\end{aligned}
$$

After substitution of Equations (11)-(16) in (9) and (10), the received signal at MS is simplified as,

$$
\begin{aligned}
& y_{\hat{X}_{1}(C M R C)}=\frac{P_{1}\left|h_{(b-m) 1}\right|^{2} X_{1}}{N_{0}}+\frac{W P_{1}\left|h_{(b-m) 4}\right|^{2} X_{1}}{N_{0}}+\frac{W P_{2}\left|h_{(r-m) 2}\right|^{2} X_{1}}{N_{0}}+N_{X_{1}(C M R C)} \\
& y_{\hat{X}_{2}(C M R C)}=\frac{P_{1}\left|h_{(b-m) 3}\right|^{2} X_{2}}{N_{0}}+\frac{W P_{1}\left|h_{(b-m) 2}\right|^{2} X_{2}}{N_{0}}+\frac{W P_{2}\left|h_{(r-m) 4}\right|^{2} X_{1}}{N_{0}}+N_{X_{2}(C M R C)}
\end{aligned}
$$

where $N_{X_{1}(C M R C)}$ and $N_{X_{2}(C M R C)}$ are the total noise at MS and described as follows:

$$
\begin{aligned}
& N_{X_{1}}=\frac{\sqrt{P_{1}} h_{(b-m) 1}^{*} n_{(b-m) 1}}{N_{0}}+\frac{\sqrt{W P_{1}} h_{(b-m) 4} n_{m 4}}{N_{0}}+\frac{\sqrt{W P_{2}} h_{(r-m) 2}^{*} n_{m 2}}{N_{0}} \\
& N_{X_{2}}=\frac{\sqrt{P_{1}} h_{(b-m) 3} n_{(b-m) 3}}{N_{0}}+\frac{\sqrt{W P_{1}} h_{(2-m) 2}^{*} n_{m 2}}{N_{0}}+\frac{\sqrt{W P_{2}} h_{(r-m) 4} n_{m 4}}{N_{0}}
\end{aligned}
$$

Finally, the total SNR at MS using C-MRC technique as follows:

$$
\begin{aligned}
\Gamma_{X_{1}} & =\frac{(1+W) P_{1}\left|h_{(b-m)}\right|^{2}+W P_{2}\left|h_{(r-m)}\right|^{2}}{N_{0}} \\
\Gamma_{X_{2}} & =\frac{(1+W) P_{1}\left|h_{(b-m)}\right|^{2}+W P_{2}\left|h_{(r-m)}\right|^{2}}{N_{0}}
\end{aligned}
$$

\section{Performance Analysis of Alamouti Decode and Forward Relaying Protocol}

\subsection{Performance Analysis of SER Asymptotic Tight Approximation}

In this paper, the performance of SER approximation for the Alamouti DF Relaying Protocol with the M-PSK modulation system is analyzed, and compared with SER upper bound. In this, the approximate SER is significantly improves at higher SNR. From the results of asymptotic tight bound approximate SER, PSO based PA is determined for the Alamouti DF Relaying Protocol.

The conditional approximate SER of the system can be follow as, 


$$
\begin{aligned}
& P_{P S K}^{h_{b-m}, h_{b-r}, h_{r-m}}=\Psi_{P S K}\left(\Gamma_{X}\right) \\
& P_{P S K}^{h_{b-m}, h_{b-r}, h_{r-m}}=\psi_{P S K}\left(\Gamma_{X}\right)=1 / \pi \int_{0}^{(M-1) \pi / M} \exp \left(-\frac{b_{p s k} \Gamma_{X}}{\sin ^{2} \theta}\right) \mathrm{d} \theta \\
& P_{P S K}^{h_{b-m}, h_{b-r}, h_{r-m}}=\psi_{P S K}\left(\Gamma_{X}\right)=1 / \pi \int_{0}^{(M-1) \pi / M} \exp \left(-\frac{b_{p s k}\left((1+W) P_{1}\left|h_{(b-m)}\right|^{2}+W P_{2}\left|h_{(r-m)}\right|^{2}\right)}{N_{0} \sin ^{2} \theta}\right) \mathrm{d} \theta
\end{aligned}
$$

where $b_{p s k}=\sin ^{2}(\pi / M)$ and $M=2^{K}$ with $K$ is even [11] [12]. In the following, the average condtional SER in Equation (25) over the rayleigh fading channels $h_{b-m}, h_{b-r}$, and $h_{r-m}$ with variances $\delta_{b-m}^{2}, \delta_{b-r}^{2}$, and $\delta_{r-m}^{2}$ respectively. Since the fading channels $h_{b-m}, h_{b-r}$, and $h_{r-m}$ are independent of each other, and

$$
\int_{0}^{\infty} \exp \left(-\frac{b_{p s k} P_{1} z}{N 0 \sin ^{2} \theta}\right) P|h|^{2}(z) \mathrm{d} z=\frac{1}{1+\frac{b_{p s k} P_{1} \delta^{h}}{N_{0} \sin ^{2} \theta}}
$$

To get the SER of the Alamouti DF Relaying Protocol with $M$-PSK modulation system can be written as,

$$
\begin{aligned}
& P_{P S K}=F_{1}\left(1+\frac{b_{p s k} P_{1} \delta_{b-m}^{2}}{N_{0} \sin ^{2} \theta}\right) F_{1}\left(1+\frac{b_{p s k} P_{1} \delta_{b-r}^{2}}{N_{0} \sin ^{2} \theta}\right)+F_{1}\left(\left(1+\frac{b_{p s k}(1+W) P_{1} \delta_{b-m}^{2}}{N_{0} \sin ^{2} \theta}\right)\left(1+\frac{b_{p s k} W P_{2} \delta_{r-m}^{2}}{N_{0} \sin ^{2} \theta}\right)\right) \\
& P_{P S K} \leq F_{1}\left(1+\frac{b_{p s k} P_{1} \delta_{b-m}^{2}}{N_{0} \sin ^{2} \theta}\right) F_{1}\left(1+\frac{b_{p s k} P_{1} \delta_{b-r}^{2}}{N_{0} \sin ^{2} \theta}\right)+F_{1}\left(\left(1+\frac{b_{p s k}(1+W) P_{1} \delta_{b-m}^{2}}{N_{0} \sin ^{2} \theta}\right)\left(1+\frac{b_{p s k} W P_{2} \delta_{r-m}^{2}}{N_{0} \sin ^{2} \theta}\right)\right)
\end{aligned}
$$

where

$$
F_{1}(x(\theta))=1 / \pi \int_{0}^{(M-1) \pi / M} \frac{1}{x(\theta)} \mathrm{d} \theta
$$

it is experiential that, substituting $\sin ^{2} \theta=1$ into the inequality in the right-hand side of Equation (27). In order to obtain maximum value in all integrands, the SER of Alamouti DF Relaying Protocol with $M$-PSK can be upper bounded as,

$$
\begin{aligned}
P_{P S K} \leq & \frac{(M-1)^{2}}{M^{2}} \frac{N_{0}^{2}}{\left(N_{0}+b_{p s k} P_{1} \delta_{b-m}^{2}\right)\left(N_{0}+b_{p s k} P_{1} \delta_{b-r}^{2}\right)} \\
& +\frac{(M-1)}{M} \frac{N_{0}^{2}}{\left(N_{0}+b_{p s k}(1+W) P_{1} \delta_{b-m}^{2}\right)\left(N_{0}+b_{p s k} W P_{2} \delta_{r-m}^{2}\right)} \\
= & \frac{(M-1) N_{0}^{2}}{M^{2}} \frac{\left[M\left(N_{0}+b_{p s k} P_{1} \delta_{b-m}^{2}\right)\left(N_{0}+b_{p s k} P_{1} \delta_{b-r}^{2}\right)\right]}{\left(N_{0}+b_{p s k}(1+W) P_{1} \delta_{b-m}^{2}\right)\left(N_{0}+b_{p s k} P_{1} \delta_{b-m}^{2}\right)\left(N_{0}+b_{p s k} P_{1} \delta_{b-r}^{2}\right)\left(N_{0}+b_{p s k} W P_{2} \delta_{r-m}^{2}\right)} \\
& +\frac{(M-1) N_{0}^{2}}{M^{2}} \frac{(M-1)\left[\left(N_{0}+b_{p s k}(1+W) P_{1} \delta_{b-m}^{2}\right)\left(N_{0}+b_{p s k} W P_{2} \delta_{r-m}^{2}\right)\right]}{\left(N_{0}+b_{p s k}(1+W) P_{1} \delta_{b-m}^{2}\right)\left(N_{0}+b_{p s k} P_{1} \delta_{b-m}^{2}\right)\left(N_{0}+b_{p s k} P_{1} \delta_{b-r}^{2}\right)\left(N_{0}+b_{p s k} W P_{2} \delta_{r-m}^{2}\right)}
\end{aligned}
$$

In the sequel, an asymptotically tight SER approximate is provided if all of the channel links $h_{b-m}, h_{b-r}$, and $h_{r-m}$ are available, i.e., $\delta_{b-m}^{2} \neq 0, \delta_{b-r}^{2} \neq 0$, and, $\delta_{r-m}^{2} \neq 0$, then when $P_{1} / N_{0}$ and $P_{2} / N_{0}$ go to infinity. According to Equation (30), let us denote the approximate SER of Alamouti DF Relaying Protocol with $M$-PSK,

$$
P_{P S K}=I_{1}\left(P_{1} / N_{0}\right)+I_{2}\left(P_{1} / N_{0}, P_{2} / N_{0}\right)
$$

where 


$$
\begin{gathered}
I_{1}(x)=F_{1}\left(1+\frac{x b_{p s k} \delta_{b-m}^{2}}{\sin ^{2} \theta}\right) F_{1}\left(1+\frac{x b_{p s k} \delta_{b-r}^{2}}{\sin ^{2} \theta}\right) \\
I_{2}(x, y)=F_{1}\left(1+\frac{(1+W) x b_{p s k} \delta_{b-m}^{2}}{\sin ^{2} \theta}\right)\left(1+\frac{W y b_{p s k} \delta_{r-m}^{2}}{\sin ^{2} \theta}\right)\left[1-F_{1}\left(1+\frac{W x b_{p s k} \delta_{b-r}^{2}}{\sin ^{2} \theta}\right)\right] \\
\lim _{x \rightarrow \infty} x^{2} I_{1}(x)=\frac{A^{2}}{b_{p s k}^{2} \delta_{b-m}^{2}, \delta_{b-r}^{2}} \\
\lim _{x, y \rightarrow \infty} x y I_{2}(x, y)=\frac{B}{b_{p s k}^{2}(1+W) W \delta_{b-m}^{2}, \delta_{r-m}^{2}} \\
A=\frac{(M-1)}{2 M}+\sin \frac{\left(\frac{2 \pi}{M}\right)}{4 \pi} \\
B=\frac{3(M-1)}{8 M}+\sin \frac{\left(\frac{2 \pi}{M}\right)}{4 \pi}-\sin \frac{\left(\frac{4 \pi}{M}\right)}{32 \pi}
\end{gathered}
$$

Therefore for large value $x$ and $y$, an asymptotically tight approximations as follows,

$$
\begin{gathered}
I_{1}(x) \approx \frac{1}{x^{2}} \frac{A^{2}}{b_{p s k}^{2} \delta_{b-m}^{2}, \delta_{b-r}^{2}} \\
I_{2}(x, y) \approx \frac{1}{x y} \frac{B}{b_{p s k}^{2}(1+W) W \delta_{b-m}^{2}, \delta_{r-m}^{2}}
\end{gathered}
$$

In this approximate, the errors become insignificant compared to the orders $\frac{1}{x y}$ when $x$ and $y$ go to infinity. Replacing $x$ and $y$ in Equations (38) and (39) with $P_{1} / N_{0}$ and $P_{2} / N_{0}$ respectively and then substituting the results into Equation (31). The asymptotically tight SER approximate with $M$-PSK modulation systems is given as,

$$
P_{P S K}=\frac{N_{0}^{2}}{b_{p s k}^{2}} \frac{1}{P_{1} \delta_{b-m}^{2}}\left(\frac{A^{2}}{P_{1} \delta_{b-r}^{2}}+\frac{B}{(1+W) W P_{2} \delta_{r-m}^{2}}\right)
$$

\subsection{Optimum Relay Selection Technique}

It is well known that the increasing number of relay in wireless communication system can achieved the diversity gain of the cooperative communication system the optimum relay selection technique such that the base station can select the optimum cooperative relay depending on the channel quality of each link [1] [11].

The optimum relay selection for Alamouti DF Relaying Protocol is given by

$$
\min \left(P_{P S K}\left(R_{1}\right), P_{P S K}\left(R_{2}\right)\right)
$$

where $R_{1}$ stands for the RS1, and $R_{2}$ stands for the RS2. From Equation (40), calculate the PSO based PA for asymptotically tight bound SER approximate $\left(P_{P S K}\right)$ for RS1 and RS2. From this, the lower approximate SER value for Alamouti DF Relaying Protocol gives the optimum relay in the cooperative communication systems $[11]$.

\subsection{Power Allocation Using Particle Swarm Optimization}

In this paper, the performance of Equal PA (EPA) and PSO based PA for Alamouti DF Relaying Protocol are analyzed and compared. Total transmitted power is $P_{T}$ then the power allocated to BS and RS as $P_{1}=p_{T} / 2$ and $P_{2}=p_{T} / 2$. PSO is optimizing technique; which gives the best optimum value for a given problem by using 
objective function. PSO contains a swarm of particles; each particle in this swarm gives a possible solution. Working of this optimizing technique is based on the population search, and gives a best solution by iteration method [27] [28].

In PSO algorithm, all particles are move towards its optimum value. For each iteration, all the particles in this swarm are updated by its position and velocity for optimization ability. In PSO, each particle maintains its position evaluated fitness and velocity. Where $r_{1}(t)$ and $r_{2}(t)$ are random variables and its elements are equally dispense in $[0,1]$. Let $P_{i}(t)$ denotes the $i$ particles position in the $m_{x}$-search space at time $t$. By updating velocity $V_{i}(t+1)$ to current position, the particles position are changed, and it is given by

$$
\begin{gathered}
V_{i}(t+1)=\omega V_{i}(t)+C_{1} r_{1}\left[y_{i}(t)+P_{i}(t)\right]+C_{2} r_{2}\left[\hat{y}(t)-P_{i}(t)\right] \\
P_{i}(t+1)=P_{i}(t)+V_{i}(t+1)
\end{gathered}
$$

Problem statement:

The SER of the systems with M-PSK modulation can be tightly approximated as

$$
P_{P S K}=\frac{N_{0}^{2}}{b_{p s k}^{2}} \frac{1}{P_{1} \delta_{b-m}^{2}}\left(\frac{A^{2}}{P_{1} \delta_{b-r}^{2}}+\frac{B}{(1+W) W P_{2} \delta_{r-m}^{2}}\right)
$$

Here the problem statement is, to minimize the $P_{P S K}$ in Equation (44)

Such that,

$$
\begin{gathered}
P_{1}=\alpha P_{T} \\
P_{2 i}=(1-\alpha) P_{T} \\
(\alpha=P A \text { factor })
\end{gathered}
$$

where

$$
\begin{gathered}
0<\alpha<1 \\
P_{1}+\sum_{i=1}^{N} P_{2 i}=P_{T}
\end{gathered}
$$

For optimum single relay selection case,

$$
P_{1}+P_{2}=P_{T}
$$

Pseudo code for particle swarm optimization

1) Initialize $\omega, C_{1}, C_{2}$

2) Allocate number of particle $\alpha$ and number of iteration

3) Generate random particles

4) Initialize random velocity $V_{i}(t)$

5) Evaluate fitness function (SER) $P_{\text {best }}$

6) If $P_{\text {best_current }}<P_{\text {best_previous }}$

$$
\begin{aligned}
& \text { Yes---update } P_{\text {best }} \\
& \text { No---end }
\end{aligned}
$$

7) If $g_{\text {best_current }}<g_{\text {best_previous }}$

Yes----update $g_{\text {best }}$

No---update velocity and position of random particle.

Continue until iteration end

From PSO algorithm, evaluate the fitness value from Equation (44) under minimum SER condition is reached. If current fitness value $\left(P_{\text {best current }}\right)$ is lesser than the previous fitness value $\left(P_{\text {best previous }}\right)$, update the minimum SER as the best fitness value $\left(P_{\text {best }}\right)$. Global best fitness value $\left(g_{\text {best }}\right)$ is calculated by comparing all the best fitness. If current global best value $\left(g_{\text {best current }}\right)$ is lesser than the previous global best fitness value $\left(g_{\text {best previous }}\right)$, update the minimum SER as the best global value $\left(g_{\text {best }}\right)$. Here 50 particles and 100 iterations are considered to achieve the optimum value [27]. Figure 3 depicts the flowchart of PSO Algorithm. 


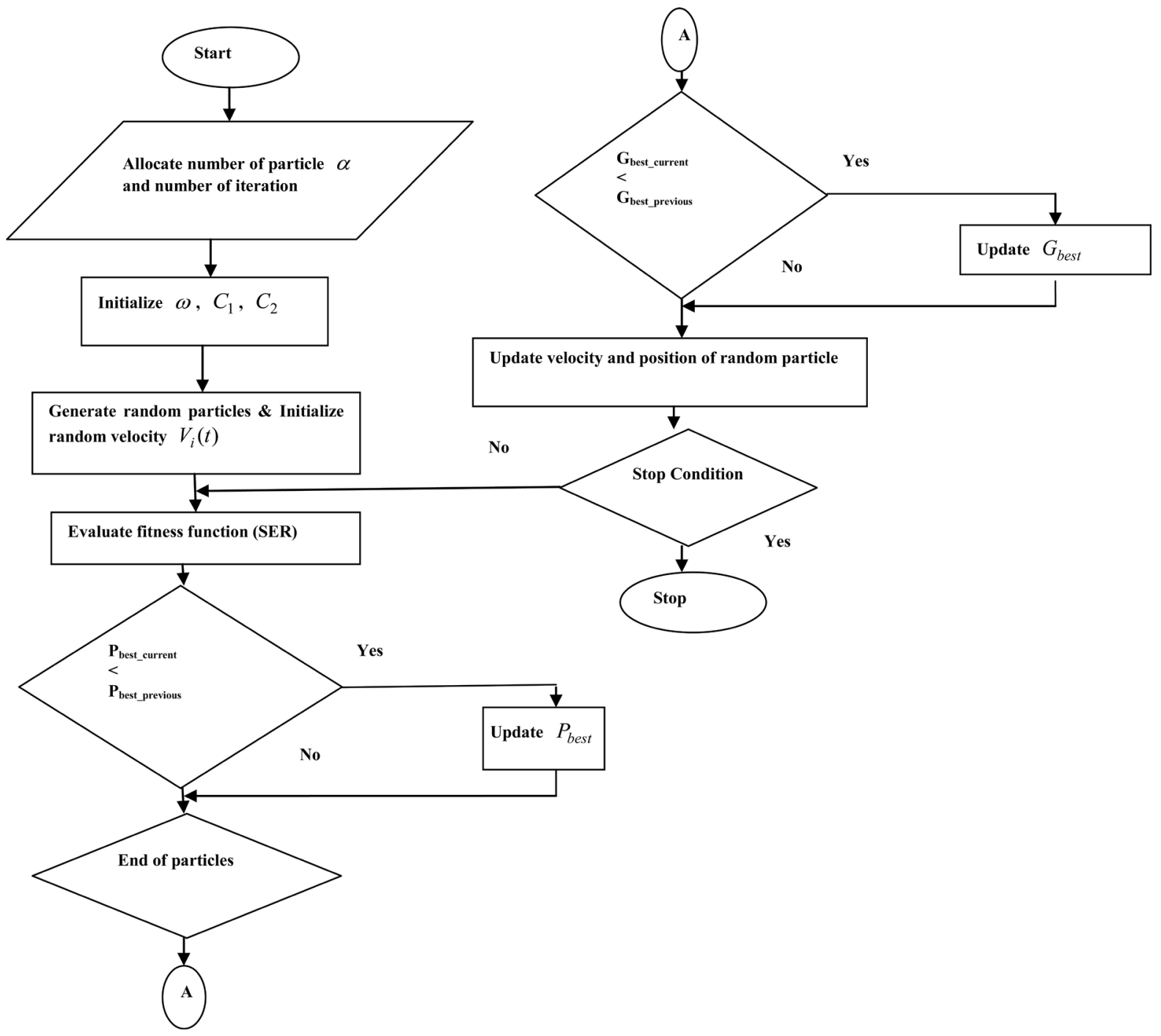

Figure 3. Flowchart of PSO Algorithm for minimizing approximate SER.

\section{Modified Cooperative Subchannel Allocation for an Alamouti DF Relaying Protocol}

In this paper, the performance analysis of a modified CSA Algorithms used in Alamouti DF Relaying Protocol for wireless multi-user OFDMA systems is discussed. The total bandwidth is $B$ and each subchannel or subcarrier is $N$ which has bandwidth of $B / N$. The noise power spectral density is $N_{0}$ level for all users and all subchannels. The Modified Throughput based CSA Algorithm enhances the throughput of the system by PSO based PA to all subchannel. In addition, the Modified Fairness based CSA Algorithm prioritize the fairness in the multi-user OFDMA systems.

\section{User Data Rate:}

The subchannel allocation matrix $A=\left[d_{i, j}\right]_{I \times J}$ specifies that the user can be allocated to the subchannels. $d_{i, j}=1$, if and only if subchannel $j$ is allocated to user $i$; or else it is zero. Any of the users not shares subchannel, so in case $d_{i, j}=1$ then $d_{l, j}=0$ for all $l \neq i$. The total transmit power is assumed as $P_{T}$ over the entire bandwidth and PSO based power allocated to the all the subchannels. In such system, the data rate for the $i^{\text {th }}$ user, $R_{i}$, is given by,

$$
R_{i}=\sum_{j}^{J} d_{i, j} r_{i, j}
$$


where $r_{i, j}$ is the SNR of the $i^{\text {th }}$ subchannel for the $j^{\text {th }}$ user and its given by,

$$
r_{i, j}=\frac{B}{2 N} \log _{2}\left(1+\frac{(1+W) P_{1}\left|h_{(b-m)}\right|^{2}}{N_{0}}+\frac{W P_{2}\left|h_{(r-m)}\right|^{2}}{N_{0}}\right)
$$

\subsection{Modified Throughput Based Subchannel Allocation}

To enhance the total throughput of the multi-user OFDMA systems, the Modified Throughput based CSA for an Alamouti DF Relaying Protocol is given by,

$$
\left[\sum_{i=1}^{I} \sum_{j=1}^{J} d_{i, j} \frac{B}{2 N} \log _{2}\left(1+\frac{(1+W) P_{1}\left|h_{(b-m)}\right|^{2}}{N_{0}}+\frac{W P_{2}\left|h_{(r-m)}\right|^{2}}{N_{0}}\right)\right]
$$

Subject to:

$$
\begin{gathered}
\max _{d_{i, j}}\left[\sum_{i=1}^{I} \sum_{j=1}^{J} d_{i, j} \frac{B}{2 N} \log _{2}\left(1+\frac{(1+W) P_{1}\left|h_{(b-m)}\right|^{2}}{N_{0}}+\frac{W P_{2}\left|h_{(r-m)}\right|^{2}}{N_{0}}\right)\right] \\
D_{1}: d_{i, j} \in\{0,1\}, \forall i, j \\
D_{2}: \sum_{i=1}^{I} d_{i, j}=1, \forall j
\end{gathered}
$$

Figure 4 shows the flowchart of Modified Throughput based Subchannel Allocation Algorithm. The constraints $D_{1}$ and $D_{2}$ guarantee that the multi user OFDMA systems are not shared all subchannels. By taking the capacity derived in Equation (52), it is experiential that the data rate is not only the function of MS subcarrier gain $\left(h_{(b-m)}\right)$, but also depends on the RS subcarrier gain $\left(h_{(r-m)}\right)$ and weighting factor $(W)$. Therefore, the channel values include the sub channels gain of RS and MS. In subchannel allocation, the selected channel

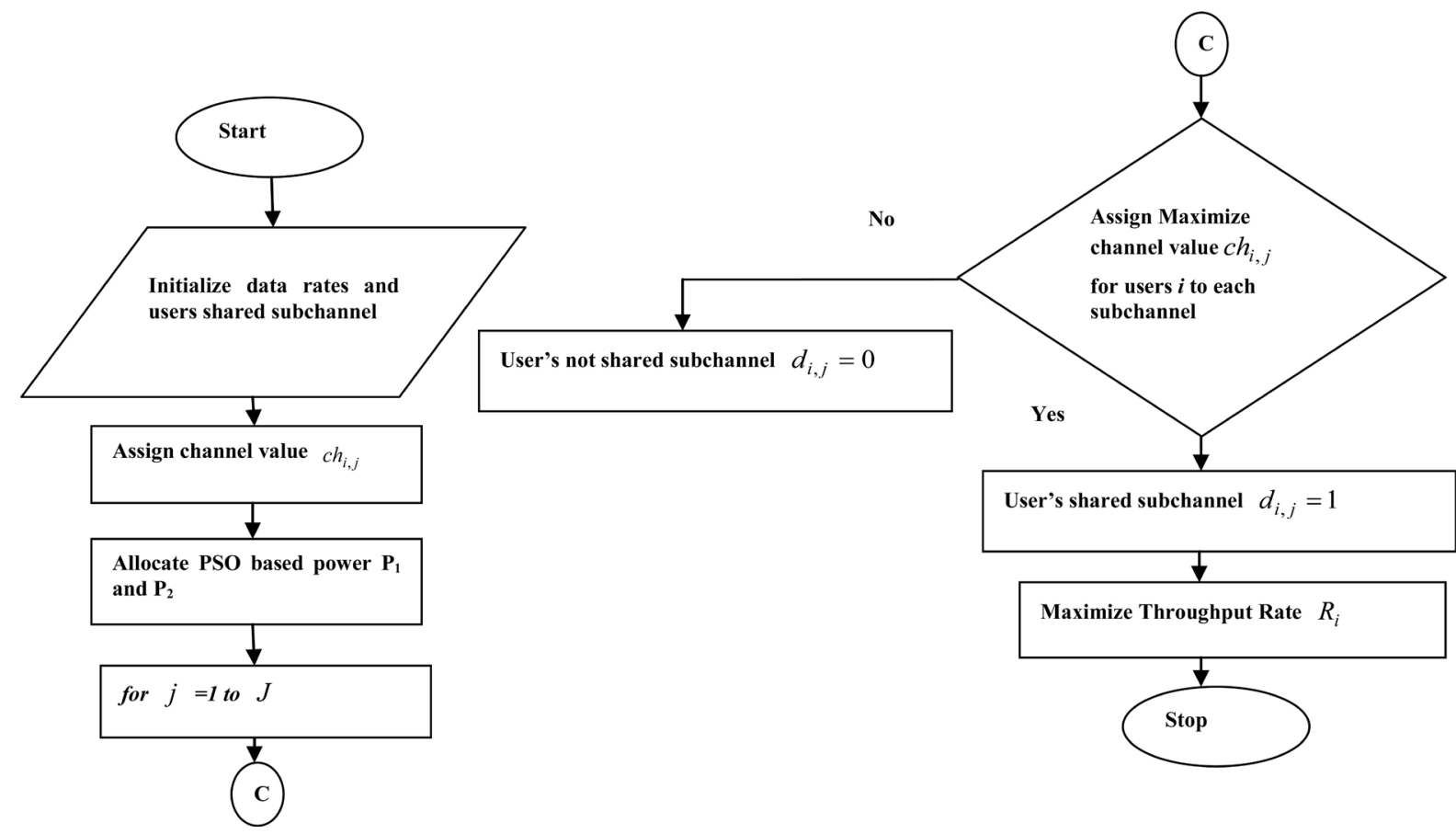

Figure 4. Flowchart of modified throughput based subchannel allocation algorithm. 
value for $i^{\text {th }}$ user as, $c h_{i, j}=(1+W) P_{1}\left|h_{(b-m)}\right|^{2}+W P_{2}\left|h_{(r-m)}\right|^{2}$.

Pseudocode For Throughput Cooperative Subchannel Allocation

1) Initialize $R_{i}=0, \forall i \& d_{i, j}=0, \forall i, j$

2) Assign channel $c h_{i, j}=(1+W) P_{1}\left|h_{(b-m)}\right|^{2}+W P_{2}\left|h_{(r-m)}\right|^{2}$

3) Allocated PSO based power to source and relay as $P_{1}$ and $P_{2}$ respectively.

4) Allocate each subchannel to the user - for $j=1$ to $J$

5) Find $i$ to maximize $c h_{i, j}$

6) Allocate subchannel to the user $d_{i, j}=1$,

7) Maximize throughput rate $R_{i}=R_{i}+\sum_{j=1}^{J} c_{i, j} r_{i, j}$,

8) end

9) end

In the first step, the channel value is considered for all the subchannels of the entire user and all the variables are initialized in the Pseudo code. In the next step, user is assigned the highest channel value for that subchannel each. For all the users, a simple two step procedure is continuing until the CSA is reached. In the OFDMA systems, thus throughput Pseudo code does not meet fairness among all users.

\subsection{Modified Fairness Based Subchannel Allocation}

To enhance the total throughput with maintaining the fairness in the multi-user OFDMA systems, by using Modified Fairness (Max-Min) based CSA for an Alamouti DF Relaying Protocol is given by:

$$
\begin{gathered}
\sum_{j=1}^{J} d_{i, j} \frac{B}{2 N} \log _{2}\left(1+\frac{(1+W) P_{1}\left|h_{(b-m)}\right|^{2}}{N_{0}}+\frac{W P_{2}\left|h_{(r-m)}\right|^{2}}{N_{0}}\right) \\
\max _{d_{i, j}} \sum_{j=1}^{J} d_{i, j} \frac{B}{2 N} \log _{2}\left(1+\frac{(1+W) P_{1}\left|h_{(b-m)}\right|^{2}}{N_{0}}+\frac{W P_{2}\left|h_{(r-m)}\right|^{2}}{N_{0}}\right) \\
D_{1}: d_{i, j} \in\{0,1\}, \forall i, j \\
D_{2}: \sum_{i=1}^{I} d_{i, j}=1, \forall j
\end{gathered}
$$

By modifying the channel values for the Fairness based CSA Pseudo code is as follows:

Pseudo Code For Fairness Cooperative Subchannel Allocation

1) Initialize $R_{i}=0, \forall i \& d_{i, j}=0, \forall i, j$

2) $A=\{1,2, \cdots, N\}$

3) Assign channel $c h_{i, j}=(1+W) P_{1}\left|h_{(b-m)}\right|^{2}+W P_{2}\left|h_{(r-m)}\right|^{2}$

4) Allocated PSO based power to source and relay as $P_{1}$ and $P_{2}$ respectively.

5) Allocate each subchannel to the user --for $i=1$ to $I$

6) (a) find j satisfying $c h j_{i, j} \geq c h_{i, m} \quad \forall m \in A \Rightarrow d_{i, j}=1$,

7) (b) Update $R_{i}$ with $R_{i}=\sum_{j=1}^{J} d_{i, j} r_{i, j}$

8) $A=A-\{j\}$

9) end while $A \neq \phi$

10) (a) find $i$ satisfying $R_{i} \leq R_{l} ; \quad \forall l=1,2, \cdots, I$

11) (b) for the found $i$ find $j$ satisfying $c h_{i, j} \geq c h_{i, m} \quad \forall m \in A \Rightarrow d_{i, j}=1$,

12) (c) update $R_{i}$ and $A$ with $i, j, R_{i}=\sum_{j=1}^{J} d_{i, j} r_{i, j}, A=A-\{j\}$

13) end

14) end 
Figure 5 shows the flowchart of Modified Fairness based Subchannel Allocation Algorithm. In the first step, the channel value is considered for all the subchannels of the entire user and all the variables are initialized in the Pseudo code. In the second step, user is assigned the channel value for that subchannel. After the entire users have been assigned to one subchannel each, in the third step priority is given to the user with the lowest data rate to choose its next subchannel. This process continues until to allocate the entire user in the systems.

\section{Simulation Result}

In this paper, the total transmitted power of $P_{T}=23 \mathrm{dBm}$ and the Channel bandwidth $B=1.4 \mathrm{MHz}$ are considered for performance evaluate of LTE downlink system.

In this the number of subchannels allocated to the users is $N=256$ and the variance of the noise is $N_{o}=10^{-3}$ assumed for analysis [29] [30].

The asymptotic performance of the Alamouti DF Relaying Protocol with 4-PSK modulation system is compared with SER upper bound and the approximation is shown in Figure 6. The normalized channel variance as 1 (i.e., $\delta_{b-m}^{2}=1, \delta_{b-r}^{2}=1, \delta_{r-m}^{2}=1$ ) and Equal Power Allocation(EPA) are assumed for simulation .In this analysis, the parameters $b=0.11, A=0.377$ and $B=0.3828$ are obtained for 4-PSK modulation system. From Figure 6, it is observed that the approximate SER is less at low SNR and significantly improves at higher SNR.

From the asymptotically tight bound approximation, the performance of Alamouti DF Relaying Protocol shows the diversity order two. Figure 7 shows the performance comparison of the PSO based PA and EPA of the Alamouti DF Relaying Protocol with 4-PSK modulation system. From Figure 7, it is observed that PSO powers ratios are $P_{1} / P=0.6670$ and $P_{2} / P=0.3333$ achieved lower approximate SER compared to EPA. The performance comparisons of the AF Relaying Protocol, DF Relaying Protocol and Alamouti DF Relaying Protocols with M-PSK modulation system are shown in Figure 8. From the simulation results, it is observed that the performance of the Alamouti DF Relaying Protocol is better than that of the DF and AF protocols at SNR of

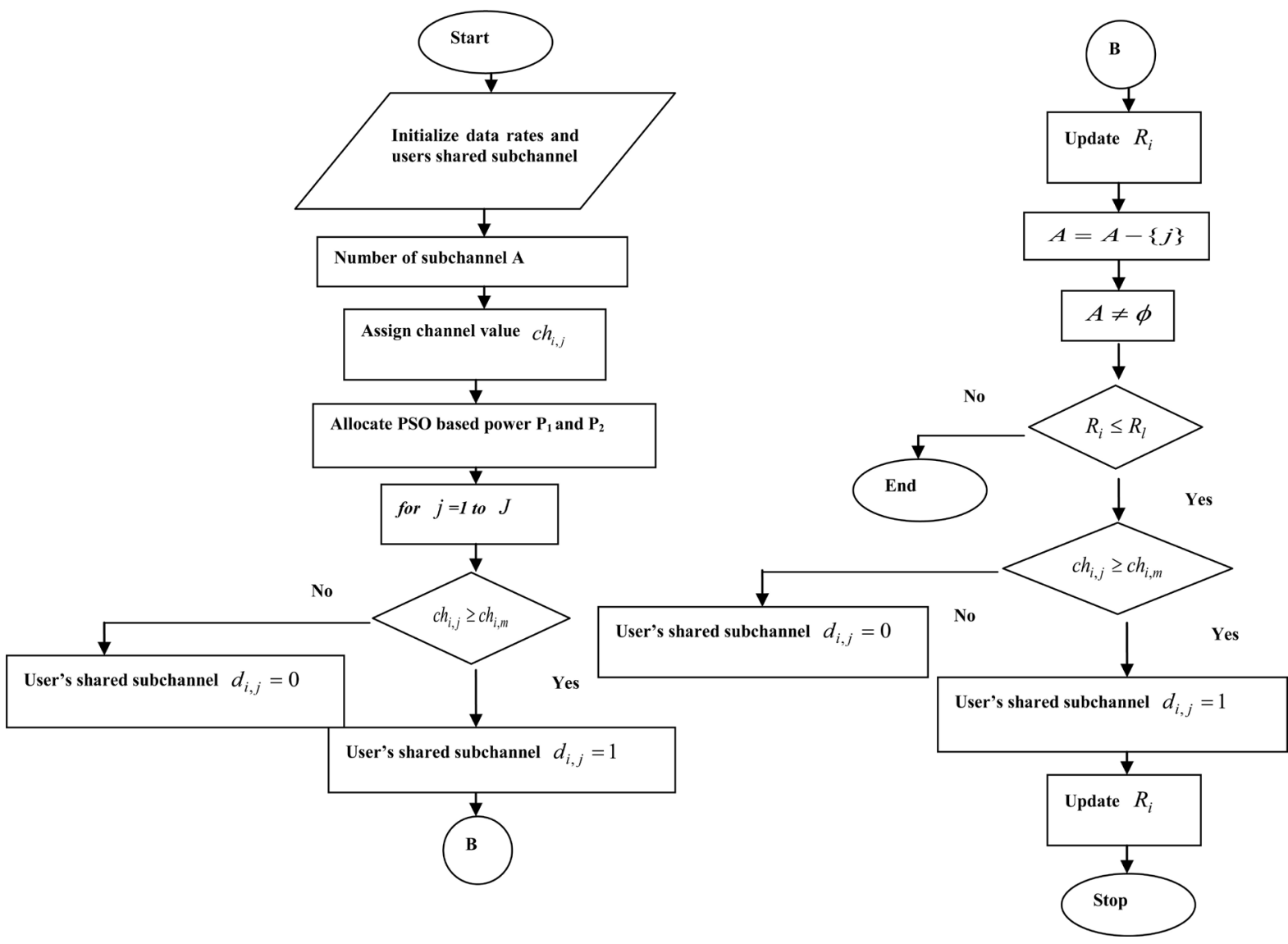




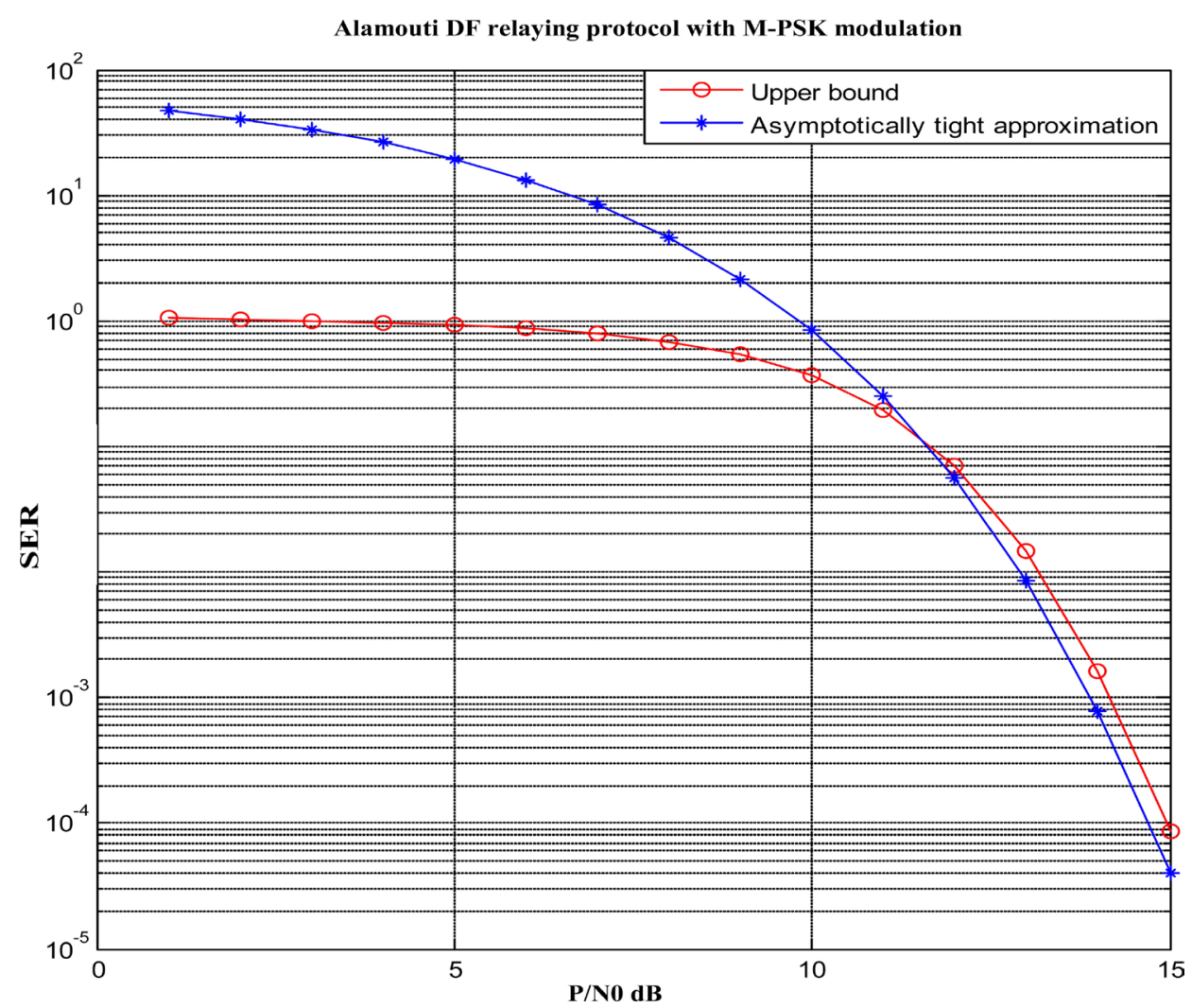

Figure 6. Performance comparison of the SER upper bound and the asymptotic tight bound approximate for the Alamouti DF Relaying Protocol with 4-PSK modulation.

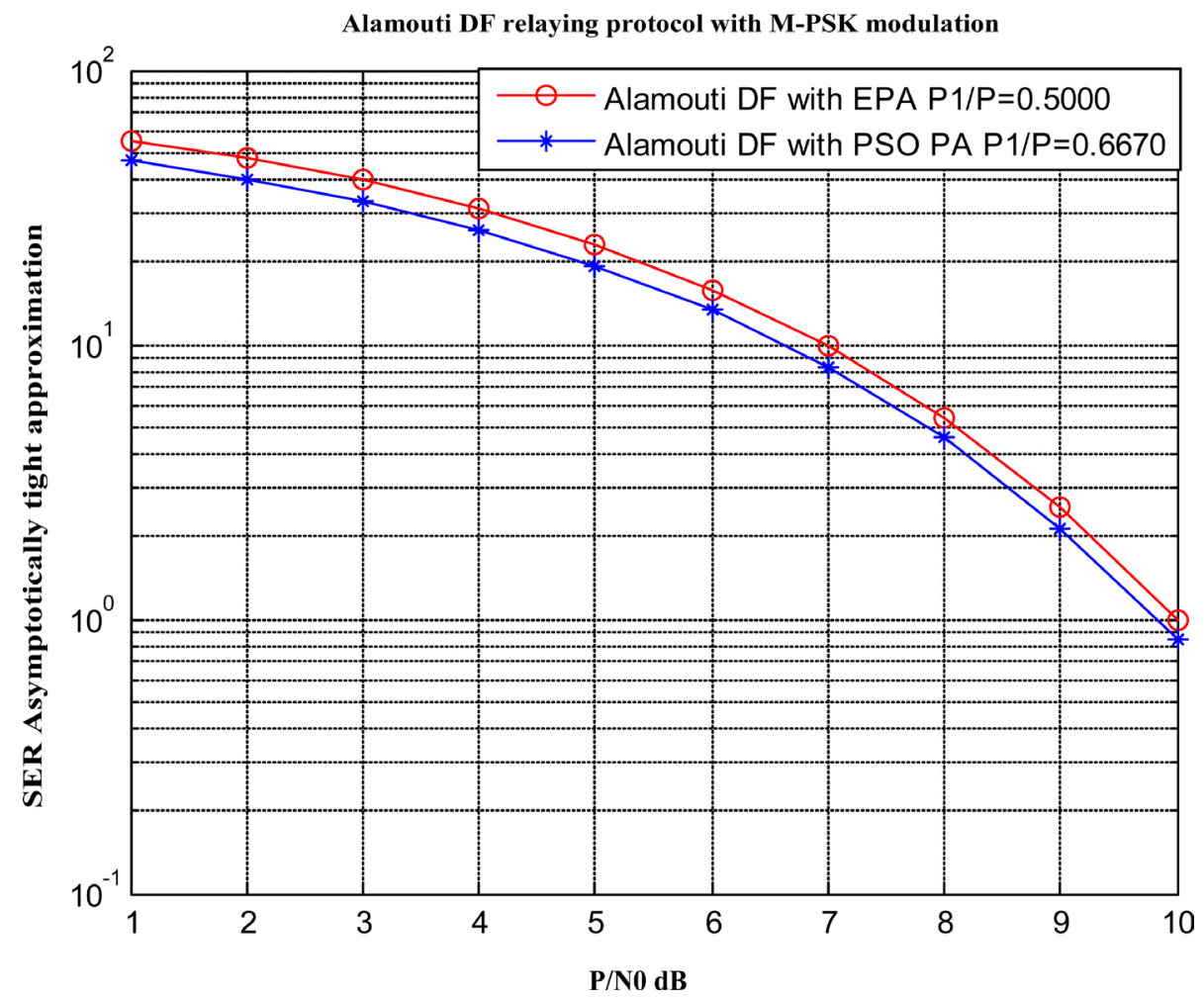

Figure 7. Performance of the Alamouti DF Relaying Protocol with 4-PSK modulation: PSO PA versus EPA. 


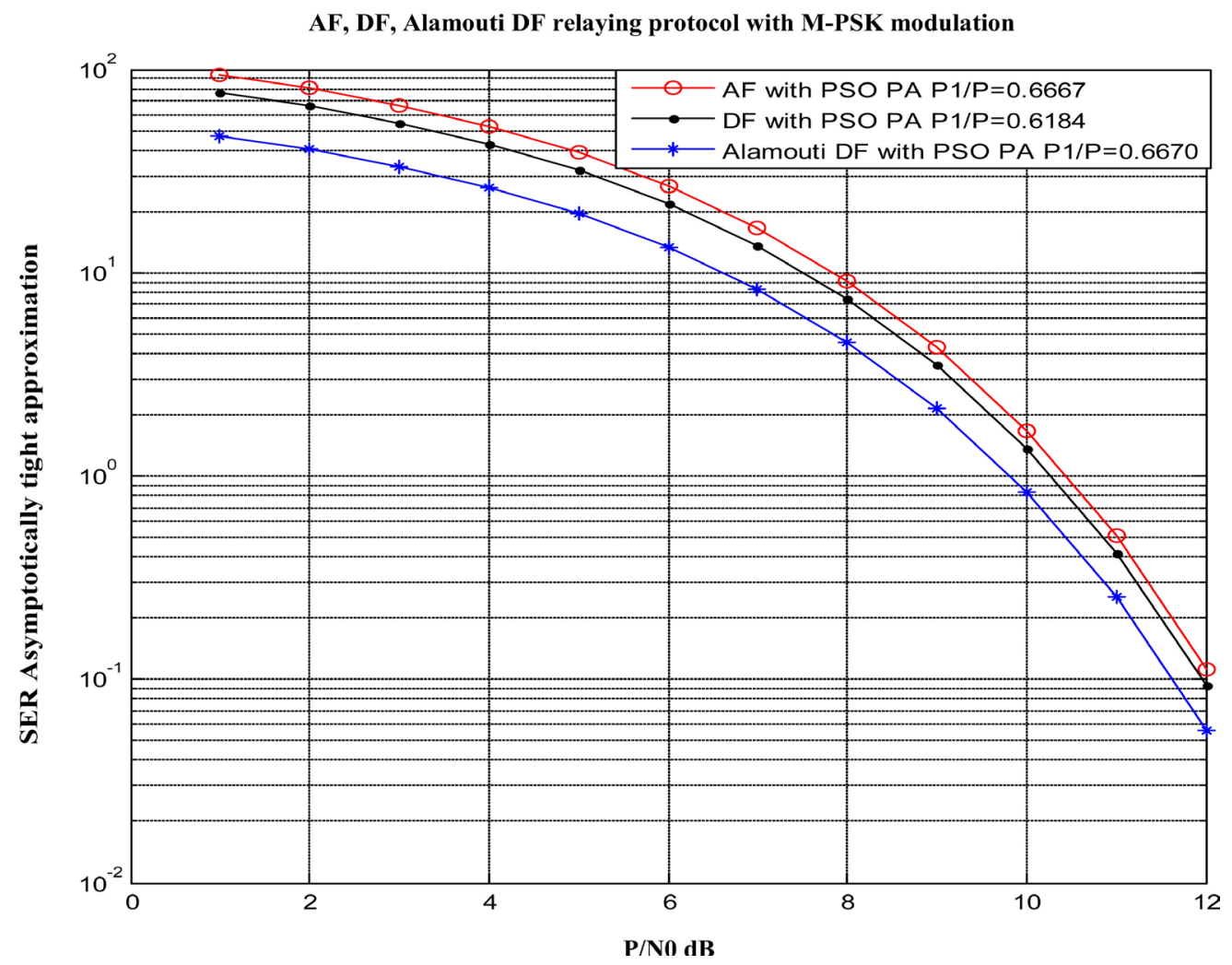

Figure 8. Performance of the Alamouti DF Relaying Protocol with 4-PSK modulation: PSO PA versus EPA.

$2 \mathrm{~dB}$ and $2.5 \mathrm{~dB}$. In the cooperative wireless communication system, the Alamouti DF Relaying Protocol is achieved improved SNR Compared to Alamouti AF Relaying Protocol, due to a decrease in the effects of additive white noise at the relay station from the decoding method. The Alamouti DF Relaying Protocol can achieve a full diversity gain with the enhanced SNR and reduced approximate SER for the base-to-mobile received signal.

From the asymptotic tight bound approximate SER, PSO power ratios $\left(P_{1} / P, P_{2} / P\right)$ for the Alamouti DF Relaying Protocol, AF Relaying Protocol and, DF Relaying Protocol are found as $(0.6667,0.3333),(0.6670$, $0.3333)$ and $(0.6184,0.3814)$ respectively.

Figure 9 shows the total transmitted power versus SER for the AF, DF and Alamouti DF Relaying Protocols with 4-PSK modulation system. The performance of three different power ratios is allocated to the $\mathrm{BS}\left(P_{1} / P\right)$ as shown in Figure 10. For $P_{1} / P=0.35$, it is observed that low power is allocated to the $\mathrm{BS}$ and high power is allocated to the RS. This is because of the SNR in the BS-to-MS link contains a weighting factor of $(1+W)$ which is higher than weighting factor $(W)$ in the RS-to-MS link.

Therefore, high power is allocated to the RS in order to maximize the total SNR. In addition for $P_{1} / P=0.65$, it is observed that high power is allocated to the BS and low power is allocated to the RS. In this case, the $\mathrm{C}$-MRC weighting factor approaches to 1 , which is the maximum value.

It is observed that the equal power is allocated to the BS and the RS for $P_{1} / P=P_{2} / P=0.5$. In this case also, the C-MRC weighting factor approaches to maximum value of 1 . The throughput modified cooperative subchannel allocation versus number of users for a wireless multi-user OFDMA system as shown in Figure 11. From this simulation results, it is observed that the Modified Throughput based Subchannel Allocation Algorithm used in Alamouti DF Relaying Protocol achieves throughput from 5.6\% to $6.3 \%$ and $21 \%$ to $33 \%$ when compared with AF Relaying Protocol and DF Relaying Protocol, respectively. In addition, the total throughput versus number of users is shown in Figure 12 using fairness based modified cooperative subchannel allocation. In this, the Modified Fairness based Subchannel Allocation Algorithm used in Alamouti DF Relaying Protocol achieves fairness among the multiuser $7.2 \%$ to $7.6 \%$ and $16.2 \%$ to $16.6 \%$ when compared with AF Relaying 
AF, DF, Alamouti DF relaying protocol with M-PSK modulation

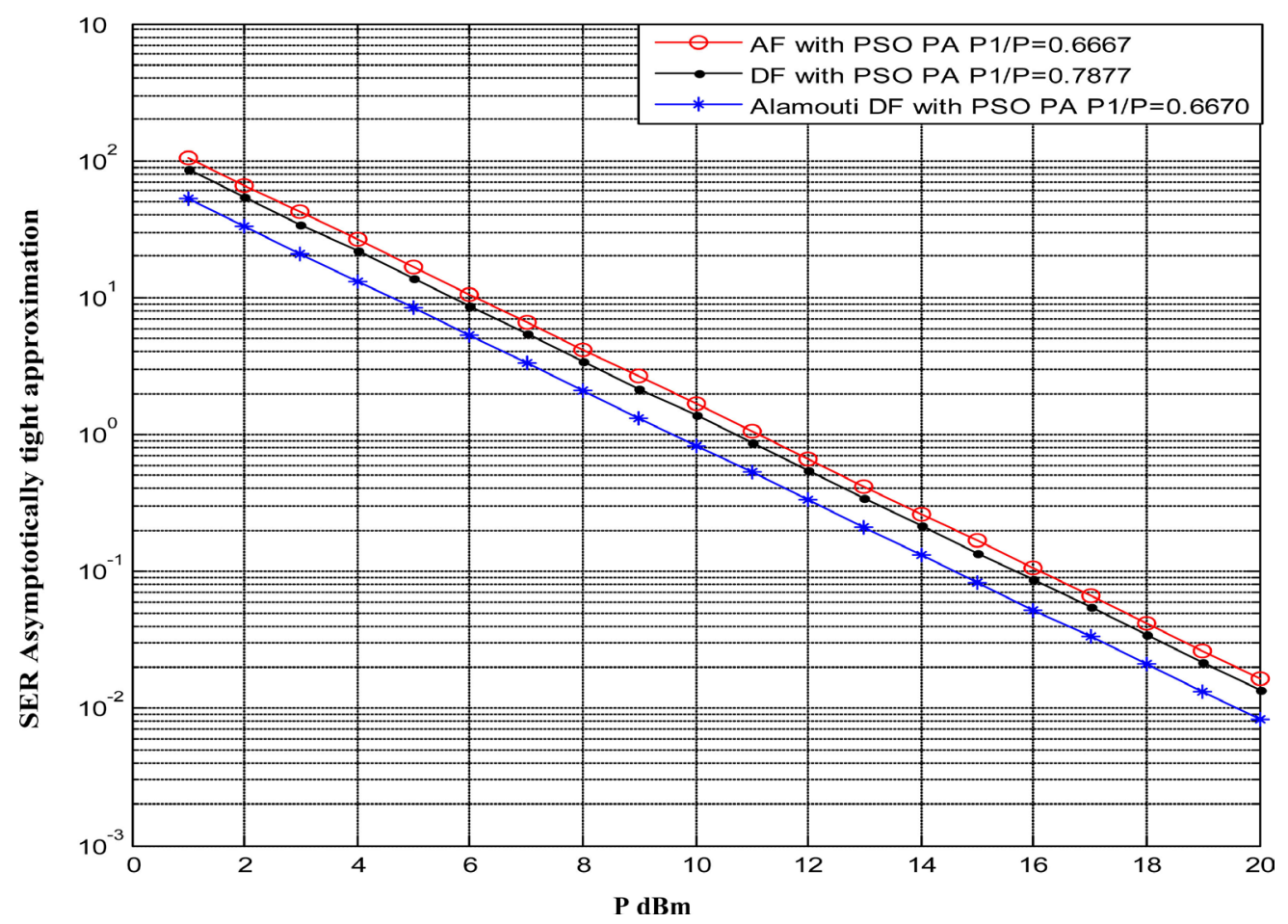

Figure 9. Performance comparison of the AF, DF, and Alamouti DF Relaying Protocols with 4-PSK modulation.

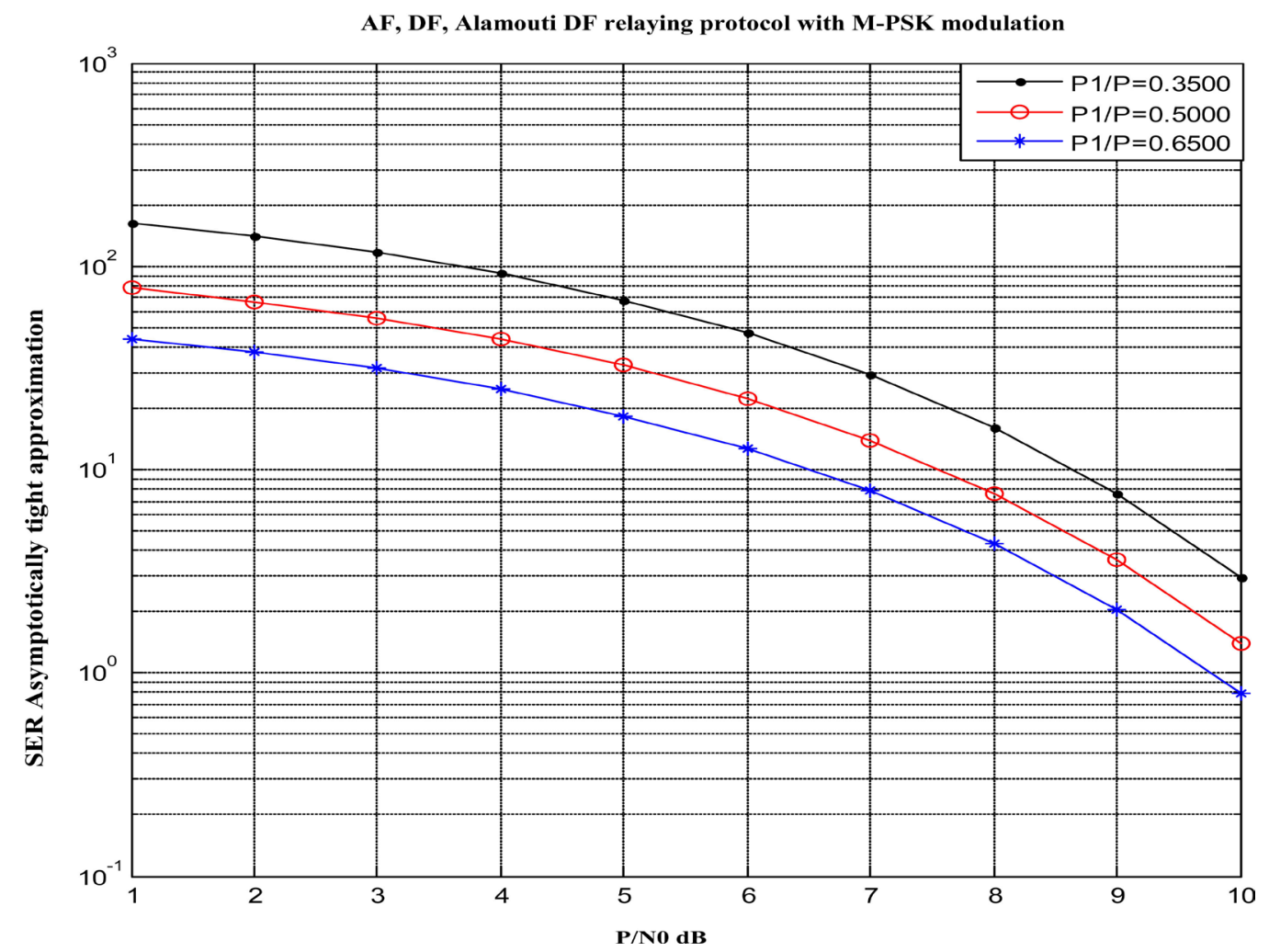

Figure 10. Performance comparison of the three different power ratios for Alamouti DF Relaying Protocol with 4-PSK modulation. 


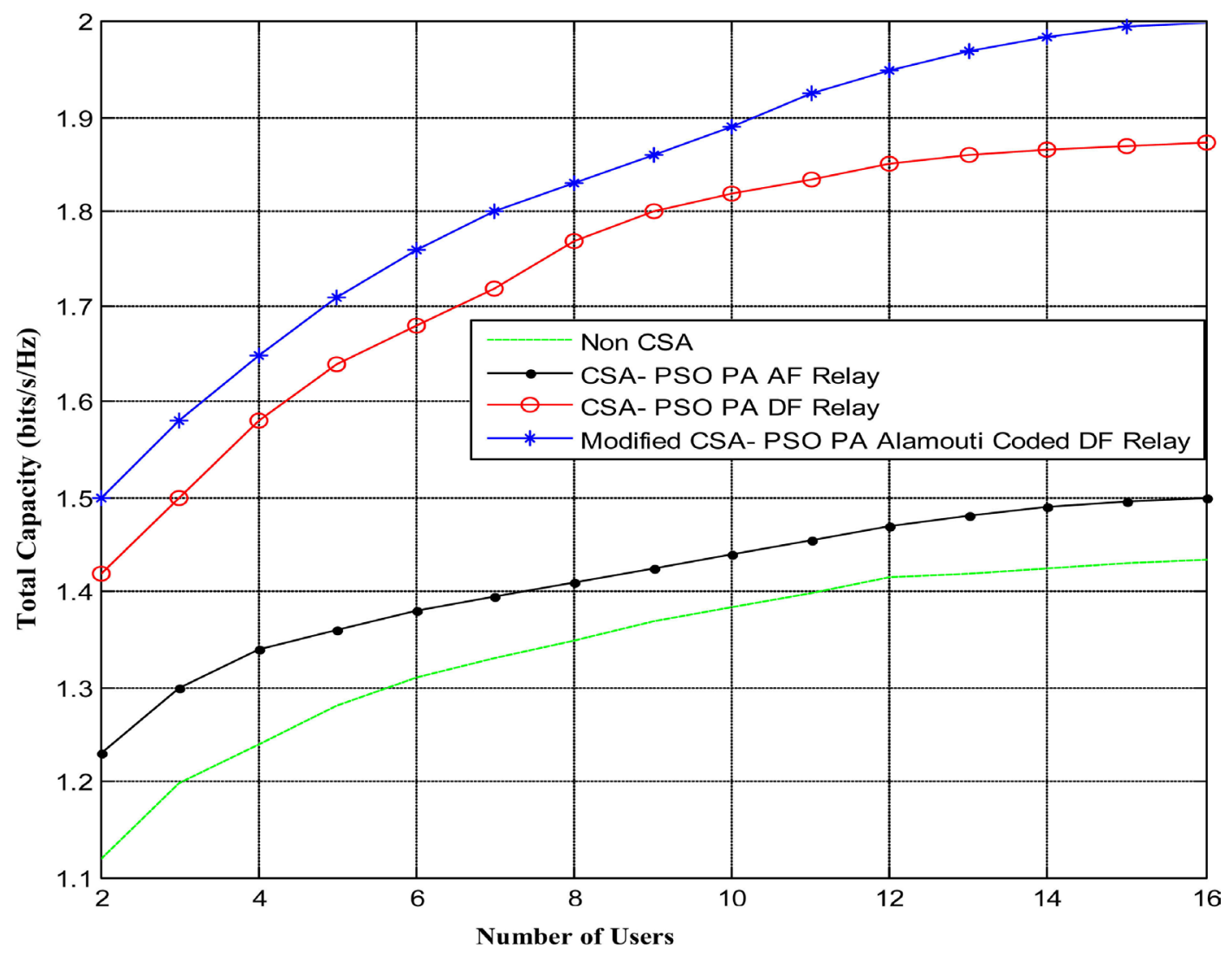

Figure 11. Total throughput for throughput oriented modified CSA algorithm.

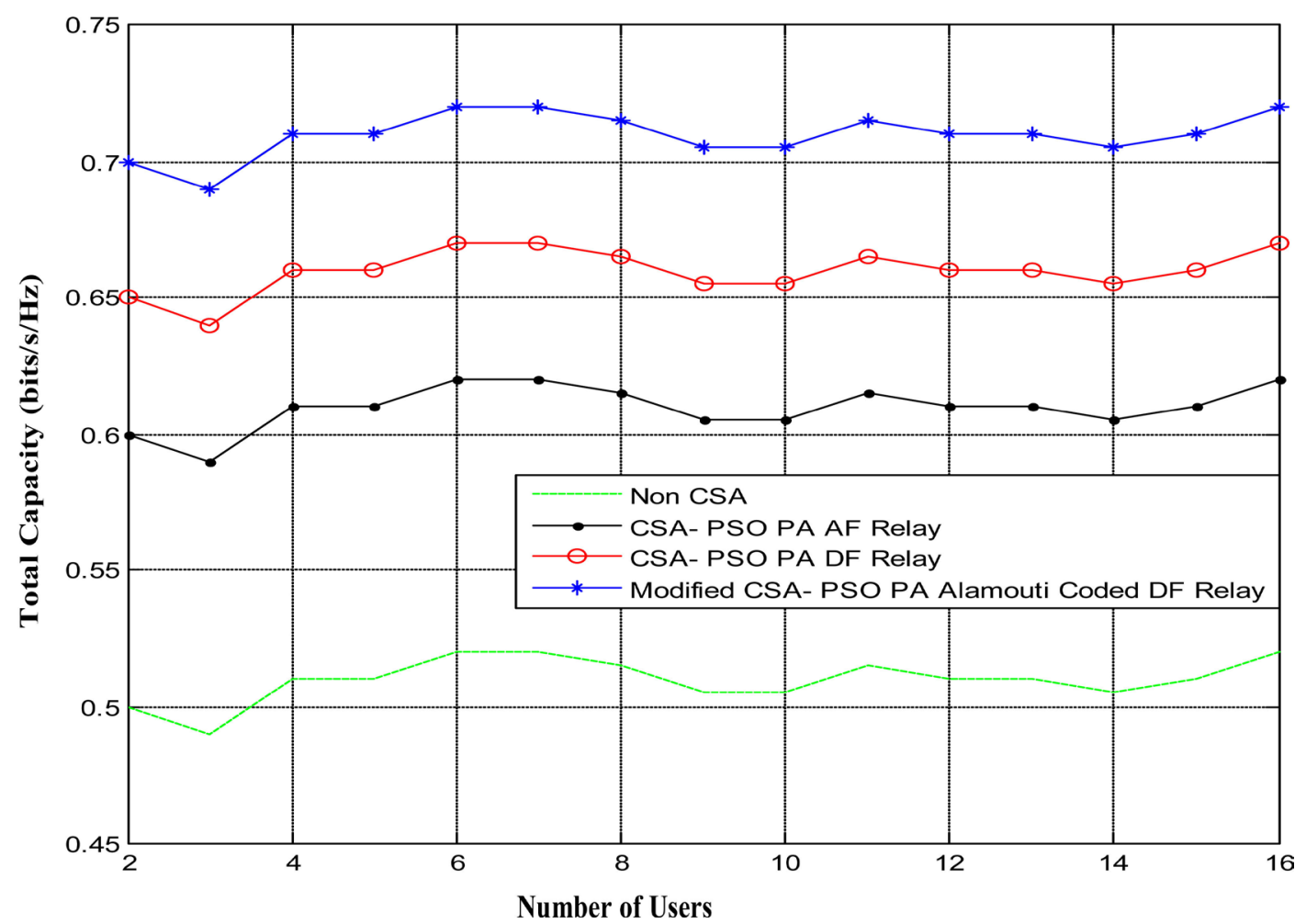

Figure 12. Total throughput for fairness oriented modified CSA algorithm. 
Protocol and DF Relaying Protocol, respectively. The modified cooperative subchannel allocation algorithm used in Alamouti DF Relaying Protocol can achieve a full diversity gain with the enhanced SNR and total throughput for wireless systems.

\section{Conclusions}

In this paper, the performance of a Modified CSA Algorithms used in Alamouti DF Relaying Protocol for wireless multi-user OFDMA systems is analyzed. In addition, the SER approximation performance analysis of Alamouti DF Relaying Protocol with PSO based PA is analyzed and compared with SER upper bound. From the simulation results, it is observed that the asymptotically approximate SER significantly improves at higher SNR region and also presents good quality match to the system performance in the reasonable-SNR region; they may not be suitable for low SNR regions.

Due to Alamouti DF Relaying Protocol, it is achieved maximum 33\% of throughput for Modified Throughput based Subchannel Allocation Algorithm. In addition, it is achieved maximum 16.6\% of fairness among the multiuser for Modified Fairness based Subchannel Allocation Algorithm. Further this research can be extended in the direction of Hybrid DF/AF Relaying Protocol for SNR improvements and throughput of the OFDMA systems.

\section{References}

[1] Laneman, J. and Wornell, G. (2003) Distributed Space-Time-Coded Protocols for Exploiting Cooperative Diversity in Wireless Networks. IEEE Transactions on Information Theory, 49, 2415-2425. http://dx.doi.org/10.1109/TIT.2003.817829

[2] Laneman, J., Tse, D. and Wornell, G. (2004) Cooperative Diversity in Wireless Networks: Efficient Protocols and Outage Behavior. IEEE Transactions on Information Theory, 50, 3062-3080. http://dx.doi.org/10.1109/TIT.2004.838089

[3] Tarokh, V., Seshadri, N. and Calderbank, A.R. (1998) Space-Time Codes for High Data Rate Wireless Communication: Performance Criterion and Code Construction. IEEE Transactions on Information Theory, 44, 744-765. http://dx.doi.org/10.1109/18.661517

[4] Alamouti, S.M. (1998) A Simple Transmit Diversity Technique for Wireless Communications. IEEE Journal on Select Areas Communications, 16, 1451-1458. http://dx.doi.org/10.1109/49.730453

[5] Tarokh, V., Jafarkhani, H. and Calderbank, A.R. (1999) Space-Time Block Codes from Orthogonal Designs. IEEE Transactions on Information Theory, 45, 1456-1467. http://dx.doi.org/10.1109/18.771146

[6] Sendonaris, A., Erkip, E. and Aazhang, B. (2003) User Cooperation Diversity-Part I: System Description. IEEE Transactions on Communications, 51, 1927-1938.

[7] Cover, T.M. and El Gamal, A.A. (2005) Capacity Theorems for the Relay Channel. IEEE Transactions on Information Theory, 25, 572-584. http://dx.doi.org/10.1109/TIT.1979.1056084

[8] Kramer, G., Gastpar, M. and Gupta, P. (2005) Cooperative Strategies and Capacity Theorems for Relay Networks. IEEE Transactions on Information Theory, 51,3037-3063. http://dx.doi.org/10.1109/TIT.2005.853304

[9] Swasdio, W., Pirak, C. and Ascheid, G. (2010) Alamouti-Coded Decode-and-Forward Protocol with Optimum Relay Selection for Cooperative Communications. 978-1-4244-6890-4/10.

[10] Swasdio, W. and Pirak, C. (2010) A Novel Alamouti-Coded Decode-and-Forward Protocol for Cooperative Communications. 978-1-4244-6890-4/10.

[11] Swasdio, W., Pirak, C., Jitapunkul, S. and Ascheid, G. (2014) Alamouti-Coded Decode-and-Forward Protocol with Optimum Relay Selection and Power Allocation for Cooperative Communications. EURASIP Journal on Wireless Communications and Networking, 2014, 112. http://dx.doi.org/10.1186/1687-1499-2014-112

[12] Su, W., Sadek, A.K. and Liu, KJ.R. (2008) Cooperative Communication Protocols in Wireless Networks: Performance Analysis and Optimum Power Allocation. Wireless Personal Communications, 44, 181-217. http://dx.doi.org/10.1007/s11277-007-9359-Z

[13] Bai, Z., Xu, Y., Dong, P., Gong, P. and Kwak, K. (2013) Particle Swarm Optimization Based Power Allocation in DF Cooperative Communications. Ubiquitous and Future Networks (ICUFN), 978-1-4673-5990-0/13.

[14] Li, F., Liu, X.-M., Luo, T. and Yue, G.-X. (2008) Optimal Power Allocation to Minimize SER for Multimode Amplify-and-Forward Cooperative Communication Systems. The Journal of China Universities of Posts and Telecommunications, 15, 14-23. http://dx.doi.org/10.1016/S1005-8885(08)60395-7 
[15] Peter, S. and Heath, R. (2009) The Future of Wimax: Multihop Relaying with IEEE 802.16j. IEEE Communications Magazine, 47, 104-111. http://dx.doi.org/10.1109/MCOM.2009.4752686

[16] Li, Y.G., Winters, J.H. and Sollenberger, N.R. (2002) MIMO-OFDM for Wireless Communications: Signal Detection with Enhanced Channel Estimation. IEEE Transactions on Communications, 50, 1471-1476. http://dx.doi.org/10.1109/tcomm.2002.802566

[17] Yang, Y., Hu, H., Xu, J. and Mao, G. (2009) Relay Technologies for WiMax and LTE-Advanced Mobile Systems. IEEE Communications Magazine, 47, 100-105. http://dx.doi.org/10.1109/MCOM.2009.5273815

[18] Jang, J. and Lee, K.B. (2003) Transmit Power Adaptation for Multiuser OFDM Systems. IEEE Journal on Selected Areas in Communications, 21, 171-178. http://dx.doi.org/10.1109/JSAC.2002.807348

[19] Yin, H. and Liu, H. (2000) An Efficient Multiuser Loading Algorithm for OFDM Based Broadband Wireless Systems. Global Telecommunications Conference, San Francisco, 27 November-1 December 2000, 103-107. http://dx.doi.org/10.1109/glocom.2000.891705

[20] Wong, C.Y. and Cheng, R.S. (1999) Multiuser OFDM with Adaptive Subcarrier, Bit and Power Allocation. IEEE Journal on Selected Areas in Communications, 17, 1747-1758. http://dx.doi.org/10.1109/49.793310

[21] Sadr, S., Anpalagan, A. and Raahemifar, K. (2009) Radio Resource Allocation Algorithms for the Downlink of Multiuser OFDM Communication Systems. IEEE Communications Surveys \& Tutorials, 11, 92-106. http://dx.doi.org/10.1109/SURV.2009.090307

[22] Sadr, S., Anpalagan, A. and Raahemifar, K. (2007) A Novel Subcarrier Allocation Algorithm for Multiuser OFDM System with Fairness: User's Perspective. 2007 IEEE 66th Vehicular Technology Conference, Baltimore, 30 September-3 October 2007, 1772-1776. http://dx.doi.org/10.1109/vetecf.2007.374

[23] Rasouli, H. and Anpalagan, A. (2011) An Opportunistic Subcarrier Allocation Algorithm Based on Cooperative Coefficient for OFDM Relaying Systems. 7th International Wireless Communications and Mobile Computing Conference (IWCMC), Istanbul, Turkey, 4-8 July 2011, 178-183.

[24] Ashourian, M., Salimian, R. and Nasab, H.M. (2013) A Low Complexity Resource Allocation Method for OFDMA System Based on Channel Gain. Wireless Personal Communications, 71, 519-529. http://dx.doi.org/10.1007/s11277-012-0826-9

[25] Alamouti, S.M. (1998) A Simple Transmit Diversity Technique for Wireless Communications. IEEE Journal on Select Areas in Communications, 16, 1451-1458. http://dx.doi.org/10.1109/49.730453

[26] Wang, T., Cano, A., Giannakis, G.B. and Laneman, J.N. (2007) High-Performance Cooperative Demodulation with Decode-and-Forward Relays. IEEE Transactions on Communications, 55, 1427-1438. http://dx.doi.org/10.1109/TCOMM.2007.900631

[27] Kennedy, J. (2010) Particle Swarm Optimization. In: Gass, S.I. and Fu, M.C., Eds., Encyclopedia of Machine Learning, Springer, New York, 760-766.

[28] Bai, Q. (2010) Analysis of Particle Swarm Optimization Algorithm. Computer \& Information Science, 3, 94-97. http://dx.doi.org/10.5539/cis.v3n1p180

[29] Gray, D. (2009) WiMAX ${ }^{\mathrm{TM}}$, HSPA+, and LTE: A Comparative Analysis.

[30] Roessler, A. and Pfeifer, G. (2010) LTE UE RF Measurements-An Introduction and Overview. 